\title{
New or noteworthy trees from Micronesia (II)
}

\author{
by \\ Ryozo Kanehira
}

Received April 16, 1932.

ANACARDIACEAE

(15) Semecarpus Kraemeri Lauterb. in EnGL. Bot. Jahrb. LVI (1921) 521 , in nota.

Arbor $15 \mathrm{~m}$ alta, usque ad $60 \mathrm{~cm}$ diametro, ramis ramulisque teretibus, glabris, lenticellatis ; ramulis incrassatis, usqus ad $2 \mathrm{~cm}$. diametro, foliis subverticellatis, coriaceis, oblanceolatis vel oblongo-oblanceolatis, ad 100 $\mathrm{cm}$. longis, $11-15 \mathrm{~cm}$. latis, in siccitate supra olivaceo-vel griseo-brunneis, subtus pallidioribus, apice obtusa acuminatis, basi acutis vel decurrentoacutis, nervis primariis utrinque circiter 20, perspicuis, parallelis, nervis secundariis inter primariis transversis, subtus prominentibus; petiolo crasso, $10 \mathrm{~mm}$. longo ; paniculis terminalibus, brevibus, partibus junioribus pubescentibus; drupis glabris, reniformis, $30 \mathrm{~mm}$. longis, hypocarpo purpurescento, $20 \mathrm{~mm}$. longo.

A large tree, $15 \mathrm{~m}$ in height, up to $60 \mathrm{~cm}$. in diameter, bark grayish, latex poisonous, the branchlets cylindric, thick, up to $2 \mathrm{~cm}$. in diameter, leaves whorled at the top of the branchlets, oblanceolate to oblong-oblanceolate, up to $100 \mathrm{~cm}$. long, $15 \mathrm{~cm}$. wide, glabrous, veins and veinlets very prominent on both surfaces; petioles stout, $10 \mathrm{~mm}$. long. Panicles terminal, drupes reniform, $30 \mathrm{~mm}$. long, hypocarpus obconical, fleshy, $20 \mathrm{~mm}$. long.

In primary forests in ravines, Tol, Truk Island, altiutde $300 \mathrm{~m}$. No. 1274! June 1931.

A very remarkable species by its thickened branchlets, very large shortpetioled leaves and large reniform drupes. It is called "panau" by the natives. The leaves and a fruit of this tree have been already collected by KraEmer in the same locality and is reported in Engt. Bot. Jahrb. LVI (1921) p. 521 by Lauterbach: "Wahrscheinlich eine S. venenosa Vuks. nahestehende neue Semecarpus-Art, welche ich vorläufig S. Kraemeri LAUTERB. nennen will." He gives a brief description in German. I here adopt his specific name and provide a more ample description.

\section{ELAEOCARPACEAE}

(16) Elaeocarpus kusaiensis KANEHIRA sp. nov.

Arbor $10 \mathrm{~m}$. alta, $60 \mathrm{~cm}$. diametro, glaber, ramis teretibus, foliis char- 
taceis, oblongis, $15-20 \mathrm{~cm}$ longis, $8-10 \mathrm{~cm}$. latis, apice obtusis, basi obtusis vel plus minusve rotundatis, margine obscure crenatis, nervis utrinque circiter 8 , distinctis; racemis axillaribus et in ramis defoliatis, usque ad 8 $\mathrm{cm}$. longis, floribus 5-meris, $8 \mathrm{~mm}$. longis, petalis lacinatis, $6 \mathrm{~mm}$. longis, $2.5 \mathrm{~cm}$. latis; fructibus ellipsoideis, usque ad $4.5 \mathrm{~cm}$. longis, $2.5 \mathrm{~cm}$. diametro.

A large glabrous tree, up to $10 \mathrm{~m}$. high, $60 \mathrm{~cm}$. in diameter. BranchIets terete, stout, $6-8 \mathrm{~mm}$. in diameter, with prominently scattered petiolar scars, nearly glabrous. Leaves numerous, crowded on the branchlets, chartaceous, oblong, $15-20 \mathrm{~cm}$. long, $8-10 \mathrm{~cm}$. wide, the apex obtuse, base obtuse or more or less rounded, obscurely crenate, lateral nerves 8 on each side of the midrib, distinct, anastomosing, the reticulations distinct, petioles $2.5-3.5 \mathrm{~cm}$. long, glabrous. Racemes axillary and on the branches below the leaves, up to $8 \mathrm{~cm}$. long, rachis pedicels and sepals sparingly appressedpubescent with pale hairs. Flowers light yellowish, 5-merous, about 8 $\mathrm{mm}$. long, their pedicels $8-15 \mathrm{~mm}$. long; sepals lanceolate, acute, $6 \mathrm{~mm}$. long, $1.5 \mathrm{~mm}$. wide, petals obovate, about $6 \mathrm{~mm}$. long, $2.5 \mathrm{~mm}$. wide, apex lacinate in the upper one fourth, stamens numerous, filaments short, ovary ovoid, densely pubescent. Fruit ellipsoid, slightly falcate, $3-4.5 \mathrm{~cm}$. long, $2-2.5 \mathrm{~cm}$. in diameter, endocarp flattened, margin irregularly dentate.

In primary forests, Mt. Hinkoln, Kusai No. 1372! July 1931.

The large oblong leaves, rather small flowers and very large fruits are characteristic features of this species. It only occurs at the higher altitudes, Kusai Island.

\section{TILTACEAE}

(17) Trichospermum Ikutai KaneHIRA sp. nov.

Trichospermum Richii (non Seem.) Volkens in Engl. Bot. Jahrb. XXXI (1901) 468.

Arbor $8 \mathrm{~m}$. alta, usque ad $40 \mathrm{~cm}$. diametro. Partibus junioribus et foliis et inflorescentiis stellato-pubescentiis; ramis ramulisque teretibus, flexuosis, nigrescentibus. Foliis distichis, alternatis, in siccitate olivaceobrunneis, oblongis ad oblongo-obovatis, usque ad $15 \mathrm{~cm}$. longis, $7.5 \mathrm{~cm}$. latis, apice obtuse acuminatis, basi inaequilateraliter cordatis, margine obscure denticulatis, leviter 3-plinerviis, nervis lateralibus 7, curvato-adscendentibus, in axillis inferioribus glandulosis. Inflorescentiis axillaribus in ramis ultimis, $10 \mathrm{~cm}$. longis, 2 - vel 3-ramosis, ramulis ultimis 3-floris, sepalis oblongis, extus dense adpresso-pubescentibus, petalis lanceolatis, obtusis; fructibus reniformis, dense adpresso-pubescentibus.

A small tree about $8 \mathrm{~m}$. in height, up to $40 \mathrm{~cm}$. in diameter, with spreading branches. The young parts, leaves and inflorescences stellate- 
tomentose. Branchas terete, flexuous, dark brown. Leaves distichous, alternate, coriaceous, dark brown when dry, sometimes paler beneath, oblong-ovate, $8-15 \mathrm{~cm}$. long, $6-7.5 \mathrm{~cm}$. wide, apex obtusely acuminate, base unequilaterally cordate, margin obscurely denticulate, scabrid on both surfaces, base somewhat 3-plinerved, with glandular axils, lateral nerves 7 on each side of the midrib, curved ascending, veins and veinlets distinct on both surfaces, petioles about $1-1.5 \mathrm{~cm}$. long, somewhat thickened at the top. Inflorescences axillary on the ultimate branches, about $10 \mathrm{~cm}$. long, 2- or 3-branched cymes, each ultimate branchlets bearing 3 umbellately arranged flowers, bracteoles deciduous, pedicels about $10 \mathrm{~mm}$. long, sepals 5, oblong, densely appressed-pubescent externally, $15 \mathrm{~mm}$. long, $5 \mathrm{~mm}$. wide, petals 5 , lanceolate, $11-13 \mathrm{~mm}$. long, $3 \mathrm{~mm}$. wide, obtuse, stamens indefinite, filaments long, filiform, ovary ovoid, densely pubescent, 2-called ; capsule kidney-shaped, $2 \mathrm{~cm}$. long, $3 \mathrm{~cm}$. wide, appressed-pubescent externally.

In thickets and secondary forests at low alt. Yap, No. 1113 (fruit) Aug. 1930, No. 1264 (flower) collected by S. Ikuta, Dec. 1930.

This tree is very common in the thickets and secondary forests on Yap, altitude about $30-50 \mathrm{~m}$. It is called "wopf" by the natives.

Volkens referred this form to Trichospermum Richii but Dr. MerRILL who compared the specimens with an isotype from Fiji, states that it is very different from the latter species.

Near Trichospermum Ledermannii, but well characterized by its large oblong-ovate, stellate-pubescent scabrid leaves with unequilaterally cordate bases and also with much larger flowers.

It is named after Shigkru Ikuta, a government assistant forester stationed at Yap who kindly assisted my explorations while I was staying there and who later sent me flowering specimens of this species.

\section{STERCULIACEAE}

(18) Heritiera longipetiolata KaneHIRA sp. nov.

Species Heritiera littoralis affinis, differt foliis ovatis, longissime petiolatis.

Arbor $15 \mathrm{~m}$. alta, usque ad $100 \mathrm{~cm}$. diametro. Partibus junioribus et subtus foliis et inflorescentiis argenteo-lepidotis. Ramis teretibus, lenticellatis. Foliis longipetiolatis, ovatis ad ovato-rhomboideis, coriaceis, usque ad $20 \mathrm{~cm}$. longis, $14 \mathrm{~cm}$. latis, apice obtusis, basi rotundatis vel truncatis plerumque subcordatis, integris, in siccitate supra brunneo-olivaceis, subtus argenteis, nitidis, nervis lateralibus 6 ad 8, curvatis, prope ad marginem arcuato-anastomosantibus, subtus distinctis; petiolo 4 ad $6 \mathrm{~cm}$. longo, glabrescenti. Fructibus subglobosis, $4 \mathrm{~cm}$. diametro, carinatis. 
A large tree up to $15 \mathrm{~m}$. high, $100 \mathrm{~cm}$. in diameter. Leaves coriaceous, ovate to ovate-rhombic, underside densely covered with silvery scales, shining, apex obtuse, the base rounded or truncate sometimes subcordate; lateral nerves 6 or 7 , curved, anastomosing, prominently elevated on the underside, reticulations distinct on both surfaces, petioles 4 to $7 \mathrm{~cm}$. long, glabrescent. Fruit subglobose, $5 \mathrm{~cm}$. long, $4 \mathrm{~cm}$. in diameter, pericarp woody, about $8 \mathrm{~mm}$. thicked, keeled.

In primary forests, Tinian, altitude about $100 \mathrm{~m}$. No. 1060 (old flower) July 1930, No. 1060 B, (fruit) collected by Mr. Y. Irobé, Feb. 1931.

A species as yet known only from Tinian where it occurs. A tree manifestly allied to Heritiera littoralis DRYand. from which it is easily distinguished by its ovate-rhombic leaves, ramarkably long petioles, and its subglobose fruits. The tree is called "ufa" by the natives and the wood is much esteemed for building purposes.

\section{GUTTIFERAE}

(19) Garcinia trukensis Kanehira sp. nov.

Arbor $15 \mathrm{~m}$. alta $40-50 \mathrm{~cm}$. diametro, ramulis crassis, angulatis, flavidoviridis ; foliis coriaceis, oblongis, usque ad $12 \mathrm{~cm}$. longis, $5.5 \mathrm{~cm}$. latis, apice latissime rostrato-acuminatis, basi subacutis, nervis lateralibus utrinque circiter 22-25, reticulis obscuris, petiolo 10-12 mm. longo ; fructibus axillaribus, fasciculatis, obovoideis, $2 \mathrm{~cm}$. longis, $1.5 \mathrm{~cm}$. latis.

A fairly large tree with straight trunk, $15 \mathrm{~m}$. high, $40-50 \mathrm{~cm}$. in dimeter. Branches dark brown, branchlets stout, angled, yellowish green ; leaves coriaceous, oblong, $9-12 \mathrm{~cm}$. long, $4.5-5.5 \mathrm{~cm}$. wide, apex broadly acuminate, acumen rostrate, base subacute, lateral nerves about 22-25 on each side of the midrib, rather distinct, parallel, anastomosing near the margin, the reticulation numerous, obscure, petioles about 10-12 mm. long.

Fruit axillary, fasciculate, obovoid, $20 \mathrm{~mm}$. long, $15 \mathrm{~mm}$. in diameter, sepals persistent, orbicular, accrescent. Flowers not seen.

In primary forests Tol, Truk Island, alt. about $450 \mathrm{~m}$. No. 1269 June 1931; Moen Truk, alt. about 250 m. No. 1705 Aug. 1931.

Very closely allied to Garcinia ponapensis LAUterb., but it is readily distinguished by its oblong leaves and obovoid fruits. The leaves seem to lack the slender diagonal lines, characteristic of Garcinia ponapensis. Nat. name: "tiamai" (Truk).

\section{MELASTOMACEAE}

(20) Astronia carolinensis $\mathrm{K}_{\text {ANEHIRA sp. nov. }}$

Arbor 5-6m. alta, ramulis et petiolis et subtus foliis ad costam nervosque plus minusve ferugineo-furfuraceis; foliis chartaceis, oblongis ad 
oblongo-obovatis, olivaceis, usque ad $12 \mathrm{~cm}$. longis, $6 \mathrm{~cm}$. latis, apice obtusis vel abrupte acutis, basi cuneato-obtusis, 3-nerviis; inflorescentiis cymosis, subglobosis, calycibus subtruncatis, glabris, petalis oblongis, $7 \mathrm{~mm}$. longis, $4 \mathrm{~mm}$. latis.

A medium-sized tree, 5-6m. high, 30-40 cm. in diameter; branchlets, petioles, lower surfaces of the leaves and inflorescences more or less ferruginous-furfuraceous, branchlets terete, stout; leaves chartaceous, oblong to oblong-obovate, olivaceous, up to $12 \mathrm{~cm}$. long, $6 \mathrm{~cm}$. wide, apex obtuse, abruptly sharp-pointed, base cuneately obtuse, nerves 3 , lateral nerves more or less ascending, distinct on the lower surface, petioles $2.5-3.5 \mathrm{~cm}$. long. Flowers terminal, cymose subglobose in outline, each branchlet bearing 3 flowers, pedicels $6-7 \mathrm{~mm}$. long, calyx cup-shaped, $5.5 \mathrm{~mm}$. long, nearly truncate or very shortly 5-lobed, glabrous externally, petals, 5 or 4, oblong, light yellowish, obtuse, $7 \mathrm{~mm}$. long, $4 \mathrm{~mm}$. wide, stamens 10 or 8 , equal in length; fruits not seen.

In primary forests at the summit of Mt. Tol, Truk, altitude about 450 m., mixed with other broad-leaved trees. Nos. 1266, 1287 June 1931; In Kusai at Mt Hinkoln (650m.), damp primary forests, No. 1380 July 1931.

This species is closely allied to Astronia palauensis KaneHIRA, but differs from it by having somewhat smaller sharply pointed leaves, subglobose inflorescences, and pale yellowish flowers.

The specimen collected in Kusai (No. 1380) has much more deeply lobed calyx than those of collected in Truk (Nos. 1266, 1287) but I think this is too minor character by which to distinguish it.

\section{SAPOTACEAE}

\section{(21) Northia Hoshinoi Kanehira sp. nov.}

Arbor trunco circiter $120 \mathrm{~cm}$. diametro, ramis ramulisque teretibus, glabris. Foliis confertis, crasse coriaceis, oblongis ad obovatis vel ovatis, in siccitate supra brunneo-olivaceis, subtus paullo pallidioribus vel subconcoloribus, apice obtusis, leviter ratusis, basi subobtusis ad acutis, nervis primariis utrinque 25 ad 35 , patulis, obscure anastomosantibus, nervis secundariis vix perspicuis, petiolo 3 ad $4 \mathrm{~cm}$. longo, glabrescenti, supra sulcato. Floribus axillaribus, solitariis vel binis, pedicellis dense adpresseque ferrugineo-pubescentibus, $1.5-2 \mathrm{~cm}$. longis, sepalis 6 , extus dense ferrugineo-pubescentibus, intus pilosis, exterioribus oblongis, obtusis, $15 \mathrm{~mm}$. longis, $7 \mathrm{~mm}$. latis, interioribus lanceolatis, $15-17 \mathrm{~mm}$. longis, $3-4 \mathrm{~mm}$. latis, corollae tubo $3-4 \mathrm{~mm}$. longo, glabro, lobis 6 , lanceolatis, acuminatis, $20 \mathrm{~mm}$. longis, $5-6 \mathrm{~mm}$. latis, filamentis $6,16 \mathrm{~mm}$. longis, villosis, antheris lanceolatis, acutis, glabris, staminoideis 6, lanceolatis, acutis, 4-5mm. longis, ovario hirsuto, 6-loculari, stylis villosis, circiter $25 \mathrm{~mm}$. longis; fructibus 
globosis, $3-4 \mathrm{~cm}$. diametro, verruculosis.

A large tree, about 10-18 m. high, up to $120 \mathrm{~cm}$. in diameter, trunk often straight, bark thick, dark brown, rough, branches spreading; leaves crowded at the tips of the branchlets, oblong to ovate, dark green, thickly coriaceous, shining, midribs impressed above, elevated beneath; flowers axillary on the ultimate branchlets, 1 or 2 in each axil, long-pedicelled, ususally nodding, about $3-3.5 \mathrm{~cm}$. wide when open, pedicels and sepals externally densely appressed ferruginous-pubescent, 6-merous, petals white, lanceolate, stamens 6 , opposite the petals, staminodes 6 , alternating with stamens, lanceolate, acute, ovary hirsute, 6-celled, each containing one ovule.

The flowers of the present plant are 6-merous and according to LAM's arrangement of the genera (Bull. Jard. Bot. Buitenz. III, 7, pp. 218 etc. 1925) it falls in Achras, but this genus is strictly American, and instead of having 10- to 12-celled ovaries, the present species has 6-celled ovaries. The only oriental genera having 6-merous flowers in this group (other than Palaquium etc., which this is not) are Manilkara and Northia, but these two genera, placed by LAm in the Mimusopeae-Maniliariae, have petals with dorsal appendages, appendages in the latter usually reduced. In this present species there is no trace of dorsal appendages, but the regetative characters are more those of Northia than Manilkara.

In thickets and forests along the road between Kolonia and Owa, Ponape, alt. 50-100 m. No. 695 (fruit) Aug. 1929, No. 1265 (flower) Dec. 1930, collected by M. Hoshino. The tree is called "kohlé" by the natives; the wood is reddish brown, hard and heavy, esteemed for making cabinets and implements. It is named in honour of Mr. Hoshino, chief of the agricultural experimental station who assisted my botanical exploration in Ponape.

\section{LOGANIACEAE}

(22) Couthovia toua Kanehira sp. nov.

Species Couthovia calophyllae affinis, differt foliis crassioribus, nervis lateralibus magis numerosis, floribus majoribus, calycibus glabris. Arbor alta, ramis ramulisque plus minusve subquadrangularis, glabris. Foliis brevipetiolatis, crasse coriaceis, in siccitate olivaceis, obovatis, usque ad $25 \mathrm{~cm}$. longis, $20 \mathrm{~cm}$. latis, apice rotundatis, basi subobtusis vel abrupte cuneato-angustatis, nervis lateralibus 9-11, parallelis, erecto-adscendentibus, nervis secundariis et reticulis subobscuris, stipulis interpetiolaribus vaginantibus. Cymis terminalibus, plerumque e basi 3-ramosis, floribus in ramulis ultimis confertis; floribus $8-9 \mathrm{~mm}$. longis, calycibus $4 \mathrm{~mm}$. longis, glabris, 5 -lobatis, lobis late ovatis, corolla $7 \mathrm{~mm}$. longa, tubo intus 
densissime longissimeque piloso, lobis ovatis, $4 \mathrm{~mm}$. longis, acutis. Fructibus aurantiacis, ellipsoideis, $45 \mathrm{~mm}$. longis, $35 \mathrm{~mm}$. latis.

A large glabrous tree, about $15 \mathrm{~m}$. high, up to $80 \mathrm{~cm}$. in diameter. Branchlets stout, grayish, subqaudrangular, internodes up to $15 \mathrm{~cm}$. long. Leaves opposite, thickly coriaceous, olivaceous when dry, paler beneath, obovate, $20-25 \mathrm{~cm}$. long, $15-20 \mathrm{~cm}$. wide, entire, apex rounded, subobtuse or abruptly narrowed toward the base, lateral nerves 9-11 on each side of the midrib, straight and parallel, ascending and anastomosing, reticulations lax, subobsolete, midrib widened toward the base, flattened or slightly sulcate, petioles $1 \mathrm{~cm}$. long, plano-convex, stipules interpetiolar, sheathing, about 6-11 mm. long, truncate. Cymes terminal, usually 3 from the uppermost of branches, $15-25 \mathrm{~cm}$. long, trichotomously branched; flowers densely crowded on the ultimate branchlets, about $8-9 \mathrm{~mm}$. long, calyx cup-shaped, $4 \mathrm{~mm}$. long, glabrous, 5-lobed, lobes broady ovate, corolla thick, woody, light yellowish, $7 \mathrm{~mm}$. long, the throat densely pilose, 5lobed, lobes ovate, $4 \mathrm{~mm}$. long, $2.5 \mathrm{~mm}$. wide, acute, anthers 5 , subsessile, stigma clavate, ovary ovoid, glabrous, 2-celled, each containing one ovule. Fruit orange-coloured, ellipsoid, $4.5 \mathrm{~cm}$. long, $3.5 \mathrm{~cm}$. in diameter, slightly pointed at both ends, pericarp thick, woody, 2 -seeded.

In primary forests, Kusai, from low to medium altitu des Nos. 1310 1391, 1405 June-July 1931.

A very handsome tree growing in primary forests, Kusai from low to medium altitudes; it is called "toua" by the natives. It is easily recognized by its large pale greenish leaves and orange-coloured pendulous fruits. The wood is much esteemed by the natives for building materials.

This species resembles Couthovia calophylla Gila et Benedict, but it is easily distinguished by its thick coriaceous leaves with numerous straight and parallel lateral nerves and also by its comparatively large flowers with glabrous calyces and ellipsoid fruits.

(23) Geniostoma kusaiense Kanehrra sp. nov.

Frutex glaber, 2 ad $4 \mathrm{~m}$. altus, ramis teretibus, ramulis distincte 4 angulatis; foliis petiolatis, chartaceis lanceolatis ad oblongo-lanceolatis, usque ad $10 \mathrm{~cm}$. longis, $3.2 \mathrm{~cm}$. latis, apice acuminatis, basi obtusis vel subaequaliter rotundatis nervis lateralibus utrinque 7 , obsolete anastomosantibus; cymis axillaribus, pedunculatis, $2 \mathrm{~cm}$. longis, pubescentibus, plerumque 3 -floris; floribus pedicellatis, $2 \mathrm{~mm}$. longis; fructibus ovoideis, acutis, $8-9 \mathrm{~mm}$. longis.

A glabrous shrub, 2 to $4 \mathrm{~m}$. high, up to $30 \mathrm{~cm}$. in diameter; branches terete, branchlets quadrangular. Leaves chartaceous, lanceolate to oblong-lanceolate, $8-10 \mathrm{~cm}$. long, $2.5-3.2 \mathrm{~cm}$. wide, apice obscurely acumi- 
nate, tip blunt, base obtuse or subequally rounded, lateral nerves 7 on each side of the midrib, obscurely anastomosing, veins distinct, petioles $3-5 \mathrm{~mm}$, long. Cymes axillary, pubescent, $2 \mathrm{~cm}$. long, pedunculate, bearing usually 3 flowers on the ultimate branchlets, pedicels slender, about 2-3 mm. long, subtended by ovate, acute $1 \mathrm{~mm}$. long bracteoles; calyx cup-shaped, $1 \mathrm{~mm}$. long, teeth triangular-ovate, acute, $0.5 \mathrm{~mm}$, long, corolla $2 \mathrm{~mm}$. long, lobes ovate, $1 \mathrm{~mm}$ : long, $0.7 \mathrm{~mm}$. wide, the throat pubescent, anthers sessile, ovary glabrous; fruit ovoid or ellipsoid, acute, $8-9 \mathrm{~mm}$. long.

In strand forests No. 1353 (fruit) also in primary forests, alt. about 500 m. No. 1362 (flower) Kusai, July 1931.

This is the fourth species of Geniostoma to be found in the Caroline Islands. A species characterized by its lanceolate leaves and very small flowers.

\section{GESNERACEAE}

(24) Cyrtandra palawensis Schltr. in ENGL. Bot. Jahrb. LVI (1921) 577.

Palau Aimiriik, 274 July 1929. In primary forests at low altitudes. Distrib. The type from Palau; endemic.

(25) Cyrtandra Urvillei C. B. Clarke in DC. Prodr. Cotin. V (1883) 251 ; SchlTr. 1. c. 576 .

Kusai Hinkoln-zan Nos. 1357, 1381, July 1931. In primary forests at the higher part of the mountain, about 580-650 m. altiutde.

Distrib. The type from Kusai; endemic.

(26) Cyrtandra ponapensis Kanehira sp. nov.

Frutex 1 ad $2 \mathrm{~m}$. altus, partibus junioribus et petiolis et subtus follis et inflorescentibus densissime ferrugineo-lanatis; foliis oppositis, subcoriaceis, ovatis ad rhomboideis, usque ad $27 \mathrm{~cm}$. longis, $15 \mathrm{~cm}$. latis, apice abrupte acutis vel obtusis, basi obtusis vel inaequilateraliter angustatis, margine irregulariter dentatis, supra olivaceis, praeter costam et nervis glabris, nervis utrinque 7 vel 8, curvato-adscendentibus, distinctis; inflorescentiis axillaribus, solitariis, breviter pedunculatis, umbellatis, 10-15 floribus, bracteis lineari-lanceolatis, ferrugineo-villosis, calycis ferrugineovillosis, $11 \mathrm{~mm}$. longis, tubo brevi, corollae tubo $9 \mathrm{~mm}$. longo, 4-lobato.

A shrub about 1-2 m. high, sparingly branched, young branchlets, petioles, underside of leaves and inflorescences very densely lanate with shining appressed ferruginous hairs; leaves opposite, subcoriaceous, ovate to rhombic, up to $27 \mathrm{~cm}$. long, $15 \mathrm{~cm}$. wide, apex abruptly acute or obtuse, base unequilaterally narrowed, margins irregularly dentate, upper surface glabrous except midrib and veins, olivaceous, the under surface densely 
ferruginous-villous, lateral nerves 7 or 8 on each side of the midrib, distinct, ascending, curved, reticulations lax, petioles $4-5 \mathrm{~cm}$. long. Inflorescences axillary, solitary, umbellate, the peduncles about $1.5 \mathrm{~cm}$. long, each bearing 5-15 flowers, their pedicels $5-6 \mathrm{~mm}$. long, the subtending bracts linear-lanceolate, ferruginous-villous, 7-8 mm. long; calyx $11 \mathrm{~mm}$. long, tube short, lobes 4 , lanceolate, acute, $3 \mathrm{~mm}$. wide, externally ferruginousvillous, corolla white, tube $9 \mathrm{~mm}$. long, lobes 4 , oblong, stamens 2 on the throat of the tube, disk about $1.5 \mathrm{~mm}$. long, glabrous, irregularly dentate, ovary and style about $9 \mathrm{~cm}$. long, glabrous, immature fruits ellipsoid, 10$18 \mathrm{~mm}$. long.

In primary forests, Niinoani-zan, altitude about $700 \mathrm{~m}$. Ponape No. 831! July 1929; in damp forests, Naanaraut-zan, altitude about $650 \mathrm{~m}$. Nos. 1606, 1642, July 1931; in forests, Tortom-zan, altitude about $600 \mathrm{~m}$. No. 1550, July 1931, Ponape.

The species is well characterized by its indumentum and its rhomboidal leaves, the nearest alliance is Cyrtandrc Urvillei from Kusai but is distinguished from its much larger and thicker leaves.

\section{RUBIACEAE}

(27) Psychotria rhombocarpa KaneHira sp. nov.

Frutex 1-2 m. altus, ramis ramulisque plus minusve quadrangulatis, glabris; foliis firmiter chartaceis, in siccitate purpureo-brunneis, supra nitidis, lanceolatis, usque ad $25 \mathrm{~cm}$. longis, $9 \mathrm{~cm}$ latis, utrinque acutis, caudato-acuminatis, leviter inaequilateralibus, nervis primariis utrinque circiter 17, curvato-adscendentibus, anastomosantibus, nervis secundariis laxis, subtus distinctis, petiolo $2-2.5 \mathrm{~cm}$. longo. Inflorescentiis paniculatis, terminalibus, ad $12 \mathrm{~cm}$. longis, puberulis, trichtome ramosis, confertis, calyx 5-dentatis, $0.7 \mathrm{~mm}$. longis, extus parce pubescentibus, corollae tubo extus glabro, $3 \mathrm{~mm}$. longo, intus ad apicem villoso, lobis oblongo-ovatis, acutis, $2 \mathrm{~mm}$. longis; fructibus rhomboideo-ovoideis, $13 \mathrm{~mm}$. longis, compressis, angulatis, seminibus plano-convexis, pentagonis., albumine aequabilo.

A shrub about 1-2 m. high, branches and branchlets somewhat quadrangular, glabrous. Leaves opposite, firmly chartaceous, chocolate-brown when dry, lanceolate, acute at both ends. Panicles terminal, puberulent, each ultimate branchlet bearing usually 3 flowers, calyx saucer-shaped in maturity, slightly pubescent, corolla white, tube constricted at the base, lobes oblong-ovate, acute. Fruit rhombic-ovoid, plano-convex, pentagonal in outline, $11 \mathrm{~mm}$. long, 9-10 $\mathrm{mm}$. wide, 2-seeded.

In damp primary forests at medium altitudes (3-400 m.) Kusai, Nos. 1321, 1333, 1403, 1439, July 1931. 
A very characteristic species with large leaves, paniculate inflorescences and also comparatively large rhombic-ovoid, somewhat angled fruits.

(28) Randia tinianensis K ANeHIRA sp. nov.

Arbor parva $6 \mathrm{~m}$. alta, $25 \mathrm{~cm}$. daim., partibus junioribus inflorescentisque puberulis exceptis glabra; foliis ovatis vel obovatis ad elliptis, usque ad $5 \mathrm{~cm}$. longis, apice obtusis vel rotundatis, basi obtusis vel abrupte angustatis, coriaceis, nitidis, in siccitate supra nigro-brunneis, nervis utrinque circiter 4, anastomosantibus; cymis axillaribus, multifloris, usque ad 3 cm. longis; fructibus depresso-globosis $6 \mathrm{~mm}$. diametro.

As mall tree, about $6 \mathrm{~m}$. high, $35 \mathrm{~cm}$. in diameter; glabrous except for the somewhat puberulent younger parts and the inflorescences; branchlets slender, somewhat compressed, grayish; leaves numerous, ovate or obovate to elliptic, $4-5 \mathrm{~cm}$. long, $2.5-3 \mathrm{~cm}$. wide, the apex obtuse or rounded, the base obtuse or abruptly narrowed, coriaceous, entire, shining, the upper surface dark brown when dry, brownish beneath, the secondary nerves 24 on each side of the midrib, anastomosing, petioles $3-5 \mathrm{~cm}$. long. Inflorescences cymose, many-flowered, $2-3 \mathrm{~cm}$. long, pedicels $2-3 \mathrm{~mm}$. long, calyx cup-shaped, $1.5 \mathrm{~mm}$. in diameter, the teeth 5 , broad, sharply pointed, corolla tube $2 \mathrm{~mm}$. long, lobes 4 or 5 , oblong, acute, $3 \mathrm{~mm}$. long, sparsely pilose at the base inside, stamens 5 , filaments $1 \mathrm{~mm}$. long, glabrous, style $4 \mathrm{~m}$. long, glabrous, stigma claviform, bifid ; fruits depressed-globose, 2celled, $6 \mathrm{~mm}$. in diameter.

Tinian Nos. 50, 950 June-July 1929, Nos. 1059, 1069, July 1930. In forests along the seashore.

A species characterized by its small leaves and densely flowered cymes.

\section{(29) Timonius megacarpus Kanehira sp. nov.}

Arbor parva 4-5m. alta, glabra, ramis ramulisque plus minusve compressis ; foliis oppositis, oblongis ad ovato-oblongis, usque ad $20 \mathrm{~cm}$. longis, $9 \mathrm{~cm}$. latis, coriaceis, apice subacutis, basi abrupte acutis vel subobtusis, nervis utrinque 7 vel 8 , perspicuis, anastomosantibus; cymis axillaribus, 2-ramosis, floribus 5-meris, calycis truncatis, fructibus globosis, 2-locularis, $3 \mathrm{~cm}$. diametro, pericarpo lepidoto.

A small tree about 4-5 m. high, glabrous throughout, branches and branchlets grayish, more or less flattened, the bark peeling off when dry. Leaves opposite, coriaceous, olivaceous when dry, oblong to ovate-oblong, 15-20 cm. long: 7-9 cm. wide, apice subacute, base abruptly acute or subobtuse, lateral nerves 7 or 8 , subparallel, anastomosing, reticulations obscure, petioles 1-2 cm. long, stipules lanceolate, acute, $7 \mathrm{~mm}$. long. Inflorescences axillary, solitary, of dichotomously branched cymes, including the flowers up to $5 \mathrm{~cm}$. long, each branch bearing 1-3 pedicelled flowers, 
subtended by broadly ovate bracts and each flower with a pair of ovate 1 mm. long bracteoles; calyx cup-shaped, glabrous, nearly truncate, $7 \mathrm{~mm}$. in diameter, $7 \mathrm{~mm}$. long, corolla $20 \mathrm{~mm}$. long, the tube appressed fulvouspubescent externally and within, lobes 5, oblong-ovate, glabrous, constricted at the base, acute, $10 \mathrm{~mm}$. long, $4 \mathrm{~mm}$. wide, stamens 5 on the throat of the corolla tube, rudimentary pistil clavate; fruit globose, $30 \mathrm{~mm}$. in diameter, pericarp lepidote, grayish brown, 2-celled, each containing 8-10 compressed pyrenes.

In thickets and primary forests Tol, Truk Island, above $400 \mathrm{~m}$. altitude. No. 1275 (송 and 우 flowers and also fruit specimens), No. 1712 (우), No. 1713 (㑒) June-Aug. 1931.

A strongly marked species; characterized by its large flowers and especially by its unusually large globose fruits. (to be continued) 